\title{
ERRORES CONCEPTUALES \\ EN EL ESTUDIO DEL EQUILIBRIO QUÍMICO: NUEVAS APORTACIONES RELACIONADAS CON LA INCORRECTA APLICACIÓN DEL PRINCIPIO DE LE CHATELIER
}

\author{
QUÍLEZ PARDO, J.' y SANJOSÉ LÓPEZ, V. ${ }^{2}$ \\ 'Instituto de Bachillerato José Ballester. València. \\ ${ }^{2}$ Didàctica de les Ciències Experimentals. Universitat de València.
}

\section{SUMMARY}

The limited usefuIness of the Le Chatelier's principle and its vague and ambiguous formulation may be some of the sources of misconceptions in chemical equilibrium. To diagnose misconceptions a written test was administered to 170 first-year students. Responses revealed that mistakes emerged through: a) misapplication and misunderstanding of the Le Chatelier's principle; $b$ ) utilization of rote-learning recall and algorithmic procedures; $c$ ) incorrect control of the variables involved and timited use of the chemical equilibrium law; $d$ ) lack of mastery of the principles of chemical equilibrium and difficulty in transfering them to new situations.

\section{INTRODUCCIÓN}

Uno de los campos de mayor estudio en investigación educativa y más extendido en las diferentes áreas de didáctica de las ciencias es el referido a la existencia de errores conceptuales en los estudiantes (Gunstone 1988). Más recientemente este estudio también se ha efectuado con profesores (Baird 1988, Banerjec 1991).

Los diferentes estudios llevados a cabo en este campo de investigación han intentado dar respuesta al menos a alguna de las sigutentes preguntas: cuáles son estos errores o dificultades, cuál puede ser su origen, en qué grado se hallan extendidos, por qué son tan resistentes al proceso de instrucción, cómo deben ser tratados y qué metodologías pueden ser más efectivas para tratar de evitarlos o superarlos.

En este trabajo intentaremos dar respuesta a algunas de estas preguntas en torno a la posible existencia de errores conceptuales debidos a la incorrecta aplicación del principio de Le Chatelier.
ERRORES CONCEPTUALES Y DIFICULTADES DE APRENDIZAJE RELACIONADOS CON EL CONCEPTO DE EQUILIBRIO QUIMICO

EI aprendizaje del concepto de equitibrio químico requiere el conocimiento previo de un gran número de conceptos relacionados con él. Ello, unido a la naturaleza abstracta del mismo, hace que sea uno de los aspectos más difíciles de enseñar (Finley et al. 1982).

Normalmente, los alumnos no tienen ideas previas sobre el cquilibrio químico al comenzar su estudio (Pozo et al. 1991), pero se ha observado que durante el desarrollo de los temas relacionados con este importante concepto aparecen una serie de dificultades de aprendizaje y de errores conceptuales (Pereira 1990, Pozo et al. 1991) que guardan relación con los siguientes aspectos:

a) Incorrecta interpretación de la doble flecha (Johnstone, et al. 1977, Cros et al. 1984). 
b) Errores relacionados con el carácter dinámico del equilibrio (Wheeler y Kass 1978, Cros et al. 1984, Hackling y Garnett 1985, Camacho y Good 1989 , Cachapuz y Maskill 1989, Bergquist y Heikkinen 1990).

c) Compartimentación del equilibrio (Johnstone et al. 1977, Furió y Ortiz 1983, Gorodetsky y Gussarsky 1986, Maskill y Cachapuz 1989).

d) Confusiones debidas a la estequiometría (Hackling y Garnett 1985, Bergquist y Heikkinen 1990).

e) Errores en el estudio de equilibrios hetcrogéneos: dificultades masa-concentración (Wheeler y Kass 1978, Furió y Ortiz 1983) y aplicación incorrecta del principio de Le Chatelier (Gorodetsky y Gussarsky 1986).

f) Dificultades con la constante de equilibrio (Wheeler y Kass 1978, Furió y Ortiz 1983, Hackling y Garnett 1985, Gorodetsky y Gussarsky 1986, Gil 1988, Camacho y Good 1989, Bergquist y Heikkinen 1990).

g) Frrores acerca del efecto de los catalizadores sobre el equilibrio (Johnstone et al. 1977, Cros et al. 1984 Hackling y Garnett 1985).

h) Errores en la predicción de la evolución de un sistema en equilibrio por un cambio de las magnitudes que lo definen: principio de Le Chatelier (Driscoll 1960, Johnstonc et al. 1977, Wheeler y Kass 1978 , Cros et al. 1984, Hackling y Garnett 1985, Gorodetsky y Gussarsky 1986, Gil 1988, Cachapuz y Maskill 1989, Bergquist y Hcikkinen 1990, Banerjee 1991).

En la mayoría de los casos, no debemos considerar estos errores conceptuales como ideas espontáneas, sino como ideas inducidas a través de la enscñanza (Johnstone et al. 1977, 1lackling y Garnett 1985). Estos errores producidos en el mismo contexto instruccional, debido, entrc otras causas, a las analogías que tanto los profesores como los libros de tex to usan para enscñar el concepto de equilibrio químico (Maskill y Cachapuz. 1989) y están asociados, en algunas ocasiones, con problemas de lenguaje (Bergquist y Heikkinen 1990), al establecer los estudiantes asociaciones y analogías con los conceptos usados en física y en la vida diaria (Gussarsky y Gorodestky 1990 )

Otra causa de las dificultades encontradas en el estudio del equilibrio químico está relacionada con la deficiente resolución de problemas (Selvaratnam y Frazer 1982), debido, en gran parte, a que los alumnos no realizan un planteamiento previo acerca de lo que hay que resolver, limitándose, en la mayoría de los casos, a establecer relaciones entre todos los datos que se proporcionan en el problema y las ecuaciones que de forma necesaria creen que deben emplear. Esta deficiente metodología puede tener en parte su origen en las estrategias didácticas de resolución de problemas que cmplean tanto los libros de tex to como los profesores. La utilización como estrategias de enseñanza de algoritmos de resolución y de reglas de tipo memorístico puede impedir a los alumnos un aprendizaje correcto de los conceplos (Nakhleh y
Mitchell 1993) y ser fuente de errores conceptuales (Bergquist y Heikkinen 1990), algunos de ellos por transmisión directa del docente. Así, se ha señalado (Quílez et al. 1993a) que una de las causas de los errores conceptuales relacionados con la aplicación del principio de Le Chatelier puede deberse a un tratamiento superficial del problema en el que no se realice un control riguroso de variables, y al empleo de cstrategias de resolución de tipo algorítmico que no cuestionan su validez y que finalmente conducen a la obtención de resuliados que en muy pocos casos son analizados. En este sentido, cl profesor debe cuestionarse cómo enseña y qué es lo que evalúa, lo cual puede facilitarle un efectivo cambio instruccional (Camacho y Good 1989).

\section{PLANTEAMIENTO DEL PROBLEMA}

Como puede deducirse por la gran atención prestada por los diferentes investigadores, uno de los aspectos más problemáticos en el estudio del equilibrio químico es la correcta comprensión del principio de Le Chatelier y su utilización adecuada por parte de los estudiantes.

Por otra parte, se ha señalado suficientemente el carácter Iimitado del principio de Le Chatelier y su formulación vaga y ambigua (Posthumus 1933, De Hecr 1957, Palacios 1958, Driscoll 1960, Wright 1969, Gold y Gold 1984, 1985, Senent y Felipe 1985, Bridgart y Kemp 1985, Solaz 1993). En estc sentido, sc ha propucsto la sustitución de la formulación tradicional del principio de Le Chatelier por una estrategia de enseñanza basada en el tratamiento termodinámico del problema (De Heer 1957, Gold y Gold 1984, 1985) en el que cl empleo de la expresión de la constantc de equilibrio evita los inconvenientes señalados (Katz 1961, Allsop y George 1984 Quílez et al. 1993). A pesar de ello, los autores de libros do texto de química general generalmente ignoran estas deficiencias, presentando el principio de Le Chatelier como un principio infalible (Qúflez et at. 1993a) y únicamente algunos libros de nivel superior (Epstein 1937, Prigogine y Defay 1954, Modell y Reid 1977 y Levine 1981 ) han discutido las limitaciones del principio de Le Chatelier.

Al deficiente planteamiento didáctico que sobre estc aspecto rcalizan muchos libros de química general y al tratamiento superficial y algorítmico en la resolución de problcmas de cquilibrio químico como estrategia de cnseñanza (Quílez et al. 1993a), debemos unir la importancia que se suele otorgar al principio de Le Chatelier en la cvaluación de los alumnos. En cste sentido, podemos recoger diferentes cuestiones propuestas en distintas pruebas de química para el acceso a la universidad:

\section{Cuestión /}

El bromuro amónico es un sólido cristalino que sc descompone en un proceso endotérmico según el siguiente equilibrio: 
$\mathrm{NH}_{4} \mathrm{Br}(\mathrm{s})=\mathrm{NH}_{3}(\mathrm{~g})+\mathrm{HBr}(\mathrm{g})$

En un recipiente de reacción en el que se ha alcanzado el equilibrio anterior:

a) Explicar si la presión del $\mathrm{HBr}(\mathrm{g})$ y la cantidad de $\mathrm{NH}_{4} \mathrm{Br}(\mathrm{s})$ aumenta, disminuye o no se modifica:

- cuando se introduce $\mathrm{NH}_{3}(\mathrm{~g})$;

- al duplicar el volumen del recipiente.

b) Deducir si el valor de la constante de equilibrio a $400^{\circ} \mathrm{C}$ será mayor, menor o igual que a $25^{\circ} \mathrm{C}$.

\section{Cuestión 2}

Para el sistema en equilibrio:

$\mathrm{Xe}(\mathrm{g})+2 \mathrm{~F}_{2}(\mathrm{~g})=\mathrm{XeF}_{4}(\mathrm{~g}) ; \Delta \mathrm{H}=-218 \mathrm{~kJ}$

predecir cuál será el efecto que tendrá sobre la composición de la mezcla en equilibrio cada una de las siguientes operaciones, dando una explicación:

a) aumento del volumen del recipiente;

b) disminución de la temperatura;

c) adición de $\mathrm{Xe}(\mathrm{g})$.

\section{Cuestión 3}

l.a síntesis del amoniaco es un proceso exotérmico.

Explicar cómo afecta al número de moles de amoniaco formados:

a) aumento de la temperatura;

b) disminución del volumen de la vasija;

c) adición de nitrógeno.

En todos los enunciados llama la atención la ausencia del control de variables que impide hacer explícita la forma en la que se realiza la perturbación.

El análisis del enunciado de las tres cuestiones lo centraremos en cada uno de los apartados que suponen la adición al sistema en equilibrio de una mayor cantidad de uno de los gases que participan en ia reacción. Así, puede parecer que lo que se pretende es que el alumno aplique el principio de Le Chatelier utilizando una regla semejante a la siguiente:

«Siempre que se añada a un sistema en equilibrio químico uno de los reactivos, el sistema evolucionará formando una mayor cantidad de productos, consumiendo parte del reactivo añadido. Si se añade uno de los productos, el efecto es el inverso al caso anterior. Los aumentos o disminuciones en la cantidad de sustancia de cada una de las especies químicas participantes se corresponderán con las variaciones en su concentración y/o presión parcial."

Este objetivo queda puesto de manifiesto de forma explícita en el siguiente ejercicio (Climent et al. 1989).

\section{Cuestión 4}

Objetivo: Utilizar el principio de Le Chatelier para predecir cómo se modifica un sistema en equilibrio cuando cambian las condiciones.

Enunciado: Dado el equilibrio:

$\mathrm{CO}(\mathrm{g})+2 \mathrm{H}_{2}(\mathrm{~g})=\mathrm{CH}_{3} \mathrm{OH}(\mathrm{g}) \quad \Delta \mathrm{H}^{0}=-18 \mathrm{~kJ}$

Cómo se modifica la cantidad de metanol en equilibrio cuando: a) se añada $\mathrm{CO}(\mathrm{g}) ; b)$ se extraiga $\mathrm{H}_{2}(\mathrm{~g})$; c) se disminuya el volumen de la vasija; d) se añada un catalizador; $e$ ) se aumente la temperatura.

Evidentemente, y aunque no es el caso que nos ocupa en estos ejemplos, la regla enunciada es incorrecta para el caso de adición (o eliminación) de un sólido en equilibrios heterogéneos. Por otro lado, y antes de continuar con la discusión de estas cuestiones, es conveniente resaltar que la citada regla se suele aplicar, basándose en la infalibilidad del principio de Le Chatelier, a sistemas que se encuentran en equilibrio. Sin embargo, antes de abordar la limitación que supone la utilización de la misma, mencionaremos el hecho de que algún libro (Mahan 1984) la utiliza como confirmación del principio de Le Chatelier para situaciones iniciales de no equilibrio, en donde el citado principio no tienc aplicación.

Siguiendo un tratamiento cyantitativo semejante al realizado por Katz. (1961), se puede demostrar que la adición de cada uno de los gases indicados producirá el efecto esperado si la misma se realiza a volumen y temperatura constantes. Sin embargo, si las variables que permanecen constantes son la presión y la temperatura, en el nuevo equilibrio existirá una mayor o menor cantidad de los mismos dependiendo de la composición inicial de los gases en equilibrio. Así, en la cuestión 1 la adición de $\mathrm{NH}_{3}(\mathrm{~g})$, a presión y temperatura constantes, producirá la descomposición del $\mathrm{NH}_{4} \mathrm{Br}(\mathrm{s})$ si, inicialmente, la fracción molar del amoniaco en el equilibrioes mayor que 0,5 . Por otro lado, se debe hacer notar que, en este caso, la producción de una mayor cantidad de gases no implica que sus respectivas presiones parciales (o sus concentraciones) también deban aumentar, al contrario de lo que indicaría una aplicación mecánica (e incorrecta) del principio de Le Chatelier (Quílez et al. 1993b). En las otras tres cuestiones formuladas, si la adicion del gas se produce a presión y temperatura constantes y su fracción molar inicial de equilibrio es superior a 0,5, la cantidad de productos será menor en el nuevo estado de 
equilibrio, al contrario de lo que en un primer tratamiento superficial se podría suponer debido a una improcedente aplicación del principio de Le Chatelier (Quñez et al. 1993a).

\section{OBJETIVOS E HIPÓTESIS}

A pesar de la abundante bibliografía que discute Ia supuesta validez del principio de Le Chatelier, ćste sigue presentándose tanto por los profesores como por los libros de texto de una forma simplificada, sin hacer referencia explícita a sus limitaciones, por to que se sucle formular como un principio infalible, capaz de predecir, en cualquier caso, la evolución de un sistema en equilibrio por la variación de las magnitudes que lo definen. Esta presentación, unida a una metodología de enseñanza que propicie un aprendizaje puramente memorístico, puede ser origen de errores conceptuales en los alumnos, fundamentalmente, por utilización del principio de Le Chatelier en situaciones donde no tiene aplicación o está limitado. Fllo implicará la no realización del pertinente control de variables y la no utilización de la expresión de la constante de equilibrio para apoyar la argumentación realizada.

En cste trabajo se estudiarán los razonamientos, explicaciones, estrategias y procedimientos que los alumnos emplean para resolver cuestiones y problemas de equilibrio químico. Fiste estudio se centrará en aquellos erro. res que pueden surgir por la incorrecta aplicación o comprensión del principio de Le Chatelier.

\section{DISEÑO EXPERIMENTAI.}

Un cuestionario consistente en cuatro cuestiones y un problema (consúltese el apéndice) se administró durante una hora a tres grupos de estudiantes de un primer curso de química general $(\mathrm{N}=170)$, unos días después de haber realizado un examen del cquilibrio químico. Tojos ellos ya habían estudiado, a un nivel más elemental, este concepto cl año anterior en el Curso de Orientación Universitaria, por lo que ya se encontraban familiarizados con muchos de los conceptos que se mancjan y, más concretamente, con el principio de Le Chatelier.

Las cuestiones diseñadas plantean situaciones en las que el principio de Le Chatelicr no tiene aplicación, se encuentra limitado, o en las que su incomprensión e intento de aplicación, fruto de un planteamiento memorístico y algorítmico, pueden provocar la predicción incorrecta del sentido en el que evoluciona un sistema en equilibrio que ha podido ser perturbado.

Analicemos de forma pormenorizada cada uno de los ítems:

ftem 1. Supone la adición a un sistema en equilibrio químico, a presión y temperatura constantes, de uno de los reactivos que participan en la reacción. Como ya se ha discutido previamente en el análisis de las cuestiones de selectividad, en este caso concreto no puede garantizarse que la adición de nitrógeno provoque un aumento en la cantidad de amoniaco en el nuevo equilibrio, al contrario de lo que ocurriria por aplicación mecánica del principio de Le Chatelier. Así, si intentamos aplicar este principio de una forma más cuidadosa, veremos que cl mismo no tiene respuesta a esta situación, ya que se producen dos efectos para los que el principio proporciona sendas predicciones contradictorias. En este caso, debemos tener en cuenta que la presión total se mantiene constante por lo que la adición de $\mathrm{N}_{2}(\mathrm{~g})$ produce un aumento en su presión parcial, micntras que la del hidrógeno disminuye, produciéndose, por tanto, dos efectos contrapuestos, de forma que el principio de Le Chatelier se encuentra limitado para predecir el resultado neto de la perturbación. A una conclusión idéntica puede llegarse si se tiene en cuenta la variación del volumen del reactor.

Item 2. Consta de dos apartados:

a) Adición de un pequeña cantidad de sólido a un equilibrio químico heterogéneo.

b) Adición a ese sistema de un gas inerte a volumen y temperatura constantes.

Un error conceptual muy extendido consiste en la aplicación del principio de Le Chatelier a cquilibrios heterogéneos por la variación en la cantidad de una de las sustancias sólidas (Whecler y Kass 1978, Gorodetsky y Gussarsky 1986). Sin embargo, muchos libros de texto no discuten de forma explícita esta situación (Quílez et al. 1993a), e incluso en algún libro de problemas (Fernández y Fidalgo 1990) aparce el crror masa-concentración (Furió y Ortiz 1983).

Por otro lado, un estudiante puede tratar de utilizar el principio de Le Chatelier para responder lo que ocurre a un sistema en equilibrio por adición al mismo de una cierta cantidad de un gas inerte, a volumen y tamperatura constantes, de la siguiente forma (Driscoll 1960): «I a adición de un gas inerte, a volumen y temperatura constantes, incrementará la presión total del sistema. Fil principio de Le Chatelier indica que el sistema se acomodará de tal forma que tienda a minimizar este aunento de presión de alguna formá. Elio puede conseguirse si la reacción que supone la formación de un menor número de moléculas se produce en una mayor extensión que en el equilibrio inicial.»

Para resolver correctamente este ítem bastará con considerar inicialmente la expresión de la constante de equilibrio y realizar los razonamientos pertinentes en función de la misma.

Item 3. Considera la posible evolución de un equilibrio químico heterogéneo por una variación de la presión total (sistema con émbolo móvil). De nuevo, la aplica- 
ción mecánica del principio de Le Chatelier y la no consideración de la expresión de la constante de equitibrio puede producir errores relacionados con el ítem 2 .

Ítem 4. Establece la adición de un gas inerte, a presión y temperatura constantes, a un sistema en equilibrio químico. Una vez más, la no consideración de la constante de equilibrio y el intento de aplicación del principio de Le Chatelier, sin el pertinente control de variables, puede producir nuevos errores. En este sentido, cabe destacar que algún texto de química de COU (Morcillo y Fernández 1990) advierte "que un cambio en el volumen del sistema, manteniendo la presión y la temperatura constantes, no modifica el equilibrio».

Mediante diferentes aproximaciones, basadas en la expresión de la constante de equilibrio (Quílez 1990), puede conseguirse la correcta resolución de este ítem.

Item 5. Se ha señalado (Allsop y George 1982) que la falta de entendimiento o de dominio de los principios del equilibrio químico, o la incapacidad de transferirlos a nuevas situaciones, pueden ser unas de las causas de las dificultades que los estudiantes encuentran en el aprendizaje de los equilibrios ácido-base, redox y de solubilidad. En este sentido, se ha diseñado este problema ácidobase. El equilibrio corresponde a una disolución acuosa de un ácido débil (ácido acéfico, HAc) y se plantea el posible efecto que sobre el mismo puede ocasionar su dilución con agua. Este efecto puede relacionarse con el correspondiente al ítem 4 (Glasstone 1946).

En este problema, la «dirección» del desplazamiento puede parecer que contradiga aquello que de una forma intuitiva se puede prever debido a un aumento del pH de la disolución (Gordus 1991). Sin embargo, el desplazamiento del equilibrio no debe analizarse en función de la variación de la acidez de la disolución, sino a partir del cambio en la cantidad de iones en la misma. Así, en este caso, la dilución con agua provoca un incremento en el número total de iones $\mathrm{H}^{+}$y Ac' en disolución, mientras que la cantidad de HAc disminuye. Este efecto no puede extrapolarse a las concentraciones. Cuando la disolución acuosa de HAc se diluye, todas las concentraciones đisminuyen. El grado de ionización del ácido acético aumenta, aunque disminuye la acidez de la disolución [H+] (Castelló y Qúlez 1992).

\section{RESULTADOS Y DISCUSIÓN}

Los resultados correspondientes al cuestionario se obtuvieron analizando la respuesta dada a cada ítem, así como la explicación correspondiente. El tipo de respuestas formuladas por los estudiantes se encuentran resumidas en la tabla 1. Se han indicado entre paréntesis los porcentajes de las respuestas que realizan predicciones acertadas y que además van acompañadas de explicaciones correctamente fundamentadas.

En el ítem 1 un $79 \%$ de los estudiantes predice un aumento en la cantidad de amoniaco. Todas estas respuestas se apoyan en el principio de Le Chatelier y entre éstas un $10 \%$ utiliza además la expresión de $\mathrm{K}_{\mathrm{c}}$ para apoyar su argumentación. Ninguna de las respuestas presentó una discusión adecuada. Algunas de las contestaciones mostraron dificultades a la hora de escribir correctamente la constante de equilibrio y en otras ocasiones se indicó que el nitrógeno añadido permanecería en exceso, sin producir desplazamiento (11\%) al no existir suficiente hidrógeno para reaccionar (reactivo limitante).

Un $73 \%$ de los estudiantes usó el principio de Le Chatelier para responder al efecto de aumento en la cantidad de sólido (ítem 2a). En algunas explicaciones se encontraron otras confusiones relacionadas con el error conceptual masa-concentración y la incorrecta expresión de la constante de equilitbrio. Así, también se señala que un aumento en la cantidad de sólido produce un aumento en su concentración y se incluye la concentración del sólido en la expresión de la constante de equilibrio. Un $24 \%$ de las respuestas realiza una predicción correcta, aunque sólo el 19\% la explica correctamente, basando su argumentación en la expresión de la constante de equilibrio. El otro $5 \%$ indica que un cambio en la cantidad de sólido no produce ningún efecto, «ya que los sólidos no reaccionan en los equilibrios heterogéneos».

Por otra parte, un $22 \%$ de las resptestas empleó un razonamiento semejante al señalado por Driscoll (1960) para predecir la posible evolución de un sistema en equilibrio químico por la adición al mismo de un gas inertc a volumen y temperatura constantes (ítcm $2 b$ ). Un $65 \%$ de las respuestas señaló que, en este caso, ningún cambio ocurriría. Sin embargo, sólo un $8 \%$ proporcionó una explicación adecuada, aunque en ningún caso sc utilizó de forma explícita la expresión de la constante de

Tabla I

Porcentajes correspondientes a posibles desplazamientos.

\begin{tabular}{|c|c|c|c|c|c|}
\hline Ítem & Derecha & No desplazam. & Izquierda & Sin predicción & No responde \\
\hline 1 & 79 & ו & 7 & - & 3 \\
\hline $2 a$ & 73 & $24(19)$ & 3 & - & - \\
\hline $2 \mathrm{~b}$ & 5 & $65(8)$ & 22 & 5 & 3 \\
\hline 3 & 24 & $40(31)$ & 16 & 16 & 4 \\
\hline 4 & $12(10)$ & 62 & 19 & 3 & 4 \\
\hline 5 & $31(9)$ & 9 & 27 & 10 & 23 \\
\hline
\end{tabular}


equilibrio. El otro $57 \%$ indicó que la adición del gas inerte no produciría ninguna variación sobre el equilibrio, ya que no reacciona con ninguna de las especies químicas que participan en el mismo.

El mejor resultado del cuestionario se obtuvo en el ítem 3 , alcanzándose un $31 \%$ de respuestas correctas. Entre ellas, un $10 \%$ basó su argumantación en la expresión de la constante de equilibrio y el otro $21 \%$ empleó el principio de Le Chatelier de forma correcta. Por otro lado, en un $9 \%$ de las respuestas se produjo una enorme variedad de explicaciones incorrectas para apoyar que no habría ningún cambio. Las respuestas que predicen un aumento en la cantidad de $\mathrm{H}_{2} \mathrm{~S}(\mathrm{~g})$ razonan apoyándose en el principio de Le Chatelier de la siguiente manera: "Una disminución en el volumen es producida por un aumento de la presión que será contrarrestada si el equilibrio se desplaza en el sentido de producción de un menor número de moléculas (incluyendo sólidos).» EI resto de las respuestas indican de forma muy distinta y variada que los cambios de volumen o presión deben ser minimizados de alguna forma.

En el ftem 4, el porcentaje de las respuestas que indican que no habrá desplazamiento $(62 \%)$ es prácticamente el mismo que el correspondiente al ítem $2 \mathrm{~b}$, indicándose de nuevo que ello es debido a que el gas inerte no reacciona. Por otra parte, y a pesar de que se señala de forma explícita que la adición se produce a presión constante, de nuevo aparece el razonamiento que indica que un aumento de la presión se compensará de forma que el equilibrio se desplace en el sentido que produzca un menor número de moléculas (19\%). Finalmente, una correcta predicción, basada en un aumento del volumen, se realizó por un $10 \%$ de las respuestas, aunque en ningún caso se utilizó la expresión de la constante de equilibrio.

En el ítem 5, un $31 \%$ de las respuestas hizo una predicción correcta. Sin embargo, la mayor parte de ellas no dio ninguna explicación. Únicamente un $9 \%$ de las respuestas realizó un razonamiento adecuado, bien basado en una correcta aplicación del principio de Le Chatelier $(6 \%)$ o en la expresión de la constante de equilibrio ( $3 \%)$. En los cuestionarios en los que en este ítem el agua aparecía de forma explícita en el equilibrio de ionización:

$$
\mathrm{CH}_{3} \mathrm{CO}_{2} \mathrm{H}(\mathrm{aq})+\mathrm{H}_{2} \mathrm{O}=\mathrm{CH}_{3} \mathrm{CO}_{2}(\mathrm{aq})+\mathrm{H}_{3} \mathrm{O}^{+}(\mathrm{aq})
$$

el porcentaje de respuestas, basadas en el principio de Le Chatelier, aumentó ligeramente (9\%).

Entre las respuestas que indican un desplazamiento «hacia la izquierda» $(27 \%)$, un $12 \%$ señala que «un descenso en la concentración de ácido acético será compensado mediante la reacción de los iones $\mathrm{H}^{+}$y Ac para producir más ácido acético (HAc)». Este error conceptual de compartimentación del equilibrio puede relacionarse con los señalados en este sentido por Johnstone y otros (1977) y Furió y Ortiz (1983). Además, un 9\% de las respuestas indicaron que la adición de agua provoca- ba un aumento de los iones $\mathrm{H}^{\star}$ o de su concentración (algunos escribieron el equilibrio de ionización acuosa del agua: $\mathrm{H}_{2} \mathrm{O} \rightleftharpoons \mathrm{H}^{+}+\mathrm{OH}^{-}$para apoyar este razonamiento). Este efecto sería minimizado por la reacción con los iones acetato, $\mathrm{Ac}^{-}$, para producir una mayor cantidad de ácido acético, HAc.

Por otro lado, entre las respuestas que indicaron que no habría desplazamiento ( $9 \%$ ), se señaló que ello era debido a que las concentraciones de todas las especies químicas disminuían en la misma proporción $(5 \%)$ o bien a que la concentración del agua no se incluye en ia expresión de la constante de equilibrio $(4 \%)$.

En lo que se refiere a la parte cuantitativa del problema, sólo en un $23 \%$ de las respuestas se abordó su realizáción y, entre éstas, la mayor parte únicamente calculó la concentración de los iones $\mathrm{H}^{+}$de la disolución de HAc $0,1 \mathrm{M}$. Únicamente un $6 \%$ calculó las concentraciones de iones $\mathrm{H}^{+}$de las disoluciones correspondientes antes y después de la dilución, aunque en ningún caso se estableció una relación entre las predicciones formuladas en el primer apartado y los cálculos realizados.

Finalmente, entre los alumnos que no dieron ninguna respuesta a este f́tem ( $23 \%$ ), algunos de ellos escribieron que no recordaban cómo se resuelve este tipo de problemas.

\section{CONCLUSIONES}

A pesar del gran número de trabajos científicos y libros que en los últimos años han sido publicados y que tratan de cuestiones surgidas de la existencia de errores conceptuales, de resolución de problemas, trabajos prácti$\cos$, cambio conceptual, metodológico y actitudinal, clima de la clase y de lo que, en definitiva, debe saber y saber hacer el profesor de ciencias, existe una gran disociación entre esta investigación educativa y la realidad escolar (Banerjec y Power 1991). Así, se hace patente que los conceptos, y más concretamente los analizados en este trabajo, son enseñados y aprendicos de forma memorística, propiciando el uso de algoritmos, los cuales suelen carecer de significado para los estudiantes. En este sentido, y desde una perspectiva constructivista, se hace necesario contemplar los prerrequisitos necesarios para abordar un concepto y las ideas previas acerca de los mismos, máxime en la introducción de un concepto tan complejo como es el de equilibrio químico (Pozo et al. 1991). Los errores detectados en este artículo con respecto a la utitización del concepto de reactivo limitante en el equilibrio químico, al manejo correcto de las leyes de los gases ideales, a la dificultad que supone la realización de un estricto control de variables, al dominio del concepto de concentración, a las dificultades masa-concentración y a otros no abordados de tipo estequiométrico y cinético, hacen necesario un cambio instruccional a la hora de introducir este importante concepto. 
Por otro lado, aunque muy relacionado con estos últimos aspectos, el empleo del principio de Le Chatelier como principal (y casi exclusiva) herramienta a la hora de predecir el efecto que produce en un sistema en equilibrio químico el cambio en las condiciones que lo definen, y la ausencia de otros razonamientos, puede hacer que se produzcan respuestas correctas, sin entender los conceptos químicos en los que se fundamentan. En este sentido, una estrategia de enseñanza de mayor rigor conceptual, basada en la leyes de la termodinámica y otra de tipo metodológico altenativa (Quílez et al. 1993) podrían ser asumidas por los profesores y autores de libros de texto.

Podemos resumir las dificultades y errores conceptuales encontrados en este trabajo en los siguientes apartados:

a) El principio de Le Chatelier se utiliza como principio infalible tanto en situaciones donde no tiene aplicación como en aquéllas en las que se encuentra limitado. La formulación que se da sobre el mismo en muchos libros

\section{REFERENCIAS BLBLIOGRÁFICAS}

ALLSOP, R.T. y GEORGE, N.H., 1982, Redox in Nuffield Advanced Chemistry, Ed. Chem., Vol. 19, pp. 57-59.

ALLSOP, R.T. y GEORGE, N.H., 1984. Le Chatelier - a redundant principle?, Ed. Chem. march, pp. 54-56.

BAIRD, J.R., 1988. Teachers in Science Education, en Fensham, P.J. (ed.), Development and Dilemmas in Science Education. (The Falmer Press: Londres).

BANERIEE, A.C., 1991. Misconceptions of students and teachers in chemical equilibrium, Int. J. Sci. Educ., Vol. 13(4), pp. 487.494

BANERJEE, A.C. y POWER, C.N., 1991. The development of modutes for the teaching of chemical equilibrium, int. I. Sci. Educ., Vol. 13(3), pp. 355-362.

BERGQUIST, W. y HEIKKINEN, H., 1990. Student Ideas Regarding Chemical Equilibrium, J. Chem. Ed., Vol. 67(12), pp. $1000-1003$.

BRIDGART, G.J. y KEMP, H.R., 1985. A Limitation on the Use of the Le Chatelier's Principle, The Australian Science Teachers' Journal, Vol. 31, pp. 60-62.

CACHAPUZ, A.F.C. y MASKILL, R., 1989. Using word association in formative classroom test: following the learning of Le Chatelier's principle, Int. J. Sci. Educ., Vol. 21(2), pp. 235-246

CAMACHO, M. y GOOD, R., 1989. Problem Solving and Chemical Equitibrium: Sticcesful versus Unsuccesful Performance, Journal of Research in Science Teaching, Vol. 26(3), pp. 251-272. de tex to y la metodología que se utiliza para su aprendizaje pueden tener una parte de responsabilidad en este aspecto. En muchas ocasiones, el intento de aplicación deI principio de Le Chatelier puede realizarse con la ayuda de la siguiente regla incorrecta:

$\mathrm{Si}$ un reactivo se añade a un sistema en equilibrio químico, el sistema «reaccionará» siempre con producción de una mayor cantidad de productos de reacción (y viceversa). Así, la adición de un gas inerte a un sistema en equilibrio no provocará ningún cambio sobre el mismo.

b) Escaso conocimiento del rango de aplicación de leyes y principios, así como dificultad de un control riguroso de variables.

c) Un uso limitado de la ley de equilibrio químico.

d) La dificultad de transferir los principios del equilibrio químico a nuevas situaciones.

CASTELLÓ, M. y QUÍLEZ, J., 1992. La construcción de la Química con ayuda delordenador. (Consellería de Educación: Valencia)

CLIMENT, M.D. etal., 1989. Quimicade COU. Una perspectiva práctica. (CESPUSA:Valencia)

CROS, D. et al., 1984. Atome, Acides-Bases. Fquilibre. Quelles idées s'en font les étudiants arrivant à l'université, Revue Française de Pédagogie, Vol. 68, pp. 49-60.

De HEER, J., 1957. The Principle of L.e Chatelier and Braun, $J$. Chem. Ed., Vol. $34(8)$, pp. $375-380$.

DRISCOLL, D.R., 1960. The Le Chatelier Principle, Australian Science Teachers Journal, Vol, 6(3), pp. 7-15.

EPSTEIN, P.E., 1937. Textbook of Thermodynamics. (John Wiley: Londres).

FERNÁNDEZ, M.R. y FIDALGO, J.A., 1990. 1000 problemas de Química General. (Everest: León)

FINLEY, F.N., STEWART, J. Y YARROCH, W.L., 1982, Teachers' perceptions of important and difficult science content, Science Education, Vol. 66(4), pp. 531-538.

FURIÓ, C.J. y ORTIZ, E., 1983. Persistencia de errores conceptuales en el estudio del equilibrio químico, Enseñanza de las Ciencias, Vol. 2(1), pp. 15-20.

GIL, V.M.S., 1988. University students' assessment of the explanatory content of justification statements, Int. J. Sci. Educ., Vol. 10(5), pp. 581-588. 
GLASSTONE, S., 1946. Textbook of Physical Chemistry. (Van Nostrand Company: Nova York).

GOLD, J. y GOLD, V., 1984. Niether Le Chatelier's nor a Principle?, Chemistry in Britain, September, pp. 802-804.

GOLD, J. y GOLD, V., 1985. Le Chatelier's Principle and the Laws of Van't Hoff, Ed. Chem., may, pp. 82-85.

GORDUS, A.D., 1991. Chemical Equilibrium, J. Chem. Educ., Vol. $68(2)$, pp. $138-140$.

GORODETSKY, M. y GUSSARSKY, E., 1986. Misconceptualization of the chemical equilibrium concept as revealed by different evaluation methods, Eur. J. Sci. Educ., Vol. 8(4), pp. 427-441.

GUNSTONE, R.F., 1988. Learners in Science Education, en Fensham, P.J. (ed.), Development and Dilemmas in Science Education. (The Falmer Press: Londres)

GUSSARSKY, E y GORODETSKY, M, 1990. On the concept "chemical equilibrium»: The associative framework, Journal of Research in Science Teaching, Vol, 27(3), pp. 197-204.

HACKLING, M.W. y GARNETT, P.J., 1985. Misconceptions of Chemical Equilibrium, Eur. J. Sci. Educ, Vol. 7(2), pp. 205-214.

IOHNSTONE, A.J., MACDONALD, J.J. y WEBB, G., 1977. Chemical equilibrium and its conceptual difficulties, $E d$. Chem., vol. 14, pp. 169-171.

KATZ, L., 1961. A Systematic Way to Avoid Le Chatelier's Principle in Chemical Reactions, J. Chem. Ed., Vol, 38(7), pp. 375-377.

LEVINE, I.N., 1981. Fisicoquímica. (McGraw-Hill:Bogotá).

MAHAN, B.H., 1984. Química. Curso Universitario. (FEI: Mejico).

MASKILL, R, y CACHAPUZ, A.F.C., 1989. Learning about the chemistry topic of equilibrium: the use of word association tests to detect developing conceptualizations, Int. J. Sci. Educ., Vol. 11(1), pp. 57-69.

MODELL, M. y REID. R.C., 1974. Thermodynamics and its Applications. (Prentice-Hall: Nueva Jersey).

MORCILLO, J. y FERNÁNDEZ, M., 1990. Química. (Anaya: Barcelona).
NAKHLEH, M.B. y MITCHELL, R.C., 1993. Concept Learning versus Problem Solving, J. Chem. Educ., Vol, 70(3), pp. 190-192.

PALACIOS, J., 1958. Termodinánica y Mecánica Estadística. (Espasa-Calpe: Madrid).

PEREIRA, M.P.B.A., 1990. Equilibrio Quimico. Dificuldades de Aprendizagem e Sugestoes Didácticas. (SPQ: Lisboa).

POSTHUMUS, K., 1933. The application of the van't Hoff-Le Chatelier-Braun Principle to Chemical Equilibra, Rec. Trav. Chim., Vol. 52, pp. 25-35.

POZO et al., 1991. Procesos cognitivos en la comprensión de la Ciencia: las ideas de los adolescentes sobre la Química. (CIDE: Madrid).

PRIGOGINE, I. y DEFAY, R. 1954. Chemical Thermodynamics. (Longmans Green: Londres).

QUÍLEZ, J. 1990. Problemas de Quínica de COU y Selectividad. (Bello: Valencia).

QUÍl.EZ, J., SOLAZ, J.J., CASTELLÓ, M. y SANJOSÉ, V., 1993a. La necesidad de un cambio metodológico en la enseñanza del equilibrio químico, Enseñanza de las Ciencias, 11(3), pp. 281-288.

QUÍL.EZ, J., DOMINGO, R. y SANJOSÉ, V., 1993b. Estudio sistemático y cuantitativo de la evolución de un sistema en equilibrio por la variación de las magnitudes que lo definen. Comunicación presentada al $I V$ Congreso de Enseñanza de las Ciencias. Barcelona.

SELVARATNAM, M. y FRAZER, M.J., 1982. Problem Solving in Chemistry. (Heinemann: Londres).

SENENT, S. y FELIPE, E., 1985. El principio de Le Chatelier cien años después, Acta Científica Compostelana, Vol. 22(1), pp. 395-411.

SOLAZ, J.J., 1993. Pourquoi continuer à aprendre le principe de Le Chatelier?, Bulletin de l'Union des Physiciens (en prensa).

WHEELER, A.E. y KASS, H., 1978. Students' misconceptions in chemical equilibrium, Science Education, Vol. 62, pp. 223-2.32

WRIGHT, P.G., 1969. A Chateierian Infidelity, Ed. Chem., Vol. 6, pp. 9 y 18. 


\section{APÉNDICE}

Razona la respuesta para cada una de las siguientes cuestiones:

1. Supongamos que partimos de $1 \mathrm{~mol}$ de $\mathrm{N}_{2}(\mathrm{~g})$ y 1 mol de $\mathrm{H}_{2}(\mathrm{~g})$ en la sintesis del amoniaco:

$\mathrm{N}_{2}(\mathrm{~g})+3 \mathrm{H}_{2}(\mathrm{~g}) \rightleftharpoons 2 \mathrm{NH}_{3}(\mathrm{~g})$

Si una vez alcanzado el equilibrio, añadimos al sistema 0,5 mol de $\mathrm{N}_{2}(g)$, a presión y temperatura constantes, indica si se desplazará el equilbrio en algún sentiơo.

2. Cuando se introduce en un reactor, inicialmente vacío, una cierta cantidad de cloruro de amonio, éste se descompone térmicamente en amoniaco y cloruro de hidrógeno, estableciéndose, a una determinada temperatura, el equilibrio representado por la siguiente ecuación:

$$
\mathrm{NH}_{4} \mathrm{Cl}(\mathrm{s})=\mathrm{NH}_{3}(\mathrm{~g})+\mathrm{HCl}(\mathrm{g})
$$

Indica cómo se podrá ver afectado el equilibrio anterior por la introducción en el recipiente (sin variar la temperatura) de una cierta cantidad de:

\section{a) $\mathrm{NH}_{4} \mathrm{Cl}(\mathrm{s})$}

b) $\mathrm{N}_{2}(\mathrm{~g})$

Notas: 1) El reactor cierra de forma hermética y es totalmente rigido.

2) El nitrógeno no reacciona con ninguna de las sustancias presentes en el equilibrio.

3. Un reactor cerrado que inicialmente contiene cantidades estequiométricas de $S(s)$ y $\mathrm{H}_{2}(\mathrm{~g})$, se calienta a una determinada temperatura, estableciéndose el siguiente equilibrio:

$$
\mathrm{S}(\mathrm{s})+\mathrm{H}_{2}(\mathrm{~g}) \rightleftharpoons \mathrm{H}_{2} \mathrm{~S}(\mathrm{~g})
$$

Indica si se desplazará el equilibrio en algún sentido en el caso de que, una vez establecido el equilibrio anterior, se reduzca el volumen de la vasija de reacción, manteniendo constante la temperatura.

4. Dado el siguiente sistema en equilibrio:

$$
\mathrm{PCl}_{5}(\mathrm{~g}) \longrightarrow \mathrm{PCl}_{3}(\mathrm{~g})+\mathrm{Cl}_{2}(\mathrm{~g})
$$

se añade, a presión y temperatura constantes, una cierta cantidad de Ne(g) (Gas inerte).

Indica si esta adición producirá el desplazamiento del equilibrio en algún sentido.

5. El equilibrio de ionización acuosa del ácido acético corresponde a la siguiente ecuación:

$$
\mathrm{CH}_{1} \mathrm{CO}_{2} \mathrm{H}(\mathrm{aq})=\mathrm{CH}_{3} \mathrm{CO} ;(\mathrm{aq})+\mathrm{H}^{+}(\mathrm{aq}) ; \mathrm{K}_{\mathrm{i}}=1.8 \times 10^{-5}
$$

Inicialmente se dispone de $100 \mathrm{~mL}$ de una disolución acuosa de ácido acético $1,0 \mathrm{M}$ a la que se le añade agua suficiente hasta obtener un volumen de $1 \mathrm{~L}$.

a) Explica el posible efecto de la adición de agua sobre el equilibrio de ionización acuosa del ácido acético.

b) Finalmente, apoya la respuesta del apartado anterior realizando los cálculos que consideres necesarios, realizando un comentrario acerca de los mismos. 